Check for updates

Cite this: RSC Adv., 2019, 9, 18344

\title{
Development of a visible light, cross-linked GelMA hydrogel containing decellularized human amniotic particles as a soft tissue replacement for oral mucosa repair
}

\author{
Qiang Zhang, (DD ab Chunyu Qian, ${ }^{a}$ Wanshu Xiao, ${ }^{a}$ Huajun Zhu, ${ }^{a}{ }^{\text {Jun }}$ Guo, ${ }^{\text {b }}$ Zili Ge ${ }^{\star a}$ \\ and Wenguo Cui ${ }^{\mathrm{C}}$
}

Early effective treatment of oral mucosal defects is the key to ensuring defect healing and functional recovery. The application of human amniotic membrane (HAM) in promoting wound healing has been shown to be safe and effective. However, amniotic membrane is thin, easy to tear and difficult to handle. Combined with the natural forces at play in the oral cavity, this has restricted the clinical applications of HAM for healing of mucosal defects. Methacrylated gelatin (GelMA) has good mechanical strength and adhesion, and can be used as a bionic repair film to attach to the damaged surface of oral mucosa, but GelMA lacks bioactive substances and cannot promote the rapid repair of oral mucosal defects. The aim of this study was to design a type of composite GelMA hydrogel mixed with decellularized human amniotic particles (dHAP) as an oral mucosa substitute, to promote regeneration of defective mucosa by stimulating rapid angiogenesis. The composite substitute GelMA-dHAP was easy to synthesize and store, and easy to operate for repair of oral mucosal defects. We show the angiogenic potential of GelMAdHAP on chick chorioallontoic membrane and the curative effect of GelMA-dHAP as a treatment in the rabbit oral mucosa defect model. In conclusion, this study confirms the effectiveness of GelMA-dHAP as an ideal soft tissue substitute for the repair of oral mucosal defects, overcoming the shortcomings of

using HAM or GelMA alone.

Received 23rd April 2019

Accepted 17th May 2019

DOI: $10.1039 / c 9 r a 03009 c$

rsc.li/rsc-advances

\section{Introduction}

Oral mucosal defects are a kind of irreversible, or reversible, damage caused by health disorders, trauma or surgery, and include cuts, tears or delayed repair of the affected parts. Wound healing is a progressive repair process of injured tissue. ${ }^{1}$ Many researchers are trying to develop an ideal biomaterial as an oral mucosal replacement that can, not only promote the repair of mucosal defects, but also has the ability to resist infection. An ideal biomaterial should have good biomechanical properties, which are similar to the oral mucosa, should promote cell adhesion, proliferation, migration and differentiation, should degrade at comparable rates to the formation of

\footnotetext{
${ }^{a}$ Department of Oral and Maxillofacial Surgery, The First Affiliated Hospital of Soochow University, Soochow University, 188 Shizi St, Suzhou, Jiangsu 215006, P. R. China.E-mail: gezl@163.com

${ }^{b}$ Department of Maxillofacial Surgery, The Affiliated Hospital of Yangzhou University, Yangzhou University, 368 Hanjiang Middle Road, Yangzhou, Jiangsu 225000, P. R. China

${ }^{c}$ Shanghai Key Laboratory for Prevention and Treatment of Bone and Joint Diseases, Shanghai Institute of Traumatology and Orthopaedics, Ruijin Hospital, Shanghai Jiao Tong University School of Medicine, 197 Ruijin 2nd Road, Shanghai 200025, P. R. China
}

new oral mucosa, and should prevent infection. ${ }^{2,3}$ So far, there is no such product, but there are some scaffold biomaterials, such as silicon-based sheets and acellular bovine collagenbased membranes (Integra ${ }^{\mathrm{TM}}$ ). Unfortunately, these materials are very expensive, so research in the field of oral mucosal regeneration has not yet met the needs of clinical application. ${ }^{4}$

Since the beginning of the 20th century, human amniotic membrane (HAM) has been successfully used for trauma and reconstruction purposes..$^{5}$ At present, HAM is still a research hotspot in tissue engineering: for example, Lai et al. used photocross-linked AM as a bioengineered limbal epithelial cell carrier. ${ }^{6}$ Many studies have also confirmed the effectiveness and safety of human amnion in wound treatment, and more than 200 cases of skin burns treated with human amniotic membrane have been reported. ${ }^{7}$

HAM is also widely used in the treatment of venous ulcers, neurovascular ulcers in diabetes, various types of posttraumatic wound dehiscence, etc. ${ }^{8-10}$ In many clinical cases, the amniotic membrane is placed on the wound and held in place with glue, sutures, or additional bandages. HAM appears to be beneficial in these clinical settings, whereas for routine clinical applications for oral mucosal repair it is a difficult material. Compared with skin substitutes, tissue-engineered 
oral mucosa must resist the natural forces of the oral cavity and should have maximum stability to keep their structure during wound healing. Although HAM has attractive biological and biochemical properties, its biodegradation rate and biomechanical properties have limited its application for regenerative medicine and tissue engineering. In order to overcome these disadvantages, HAM has been modified to adjust its performance by chemical cross-linking or innovative stratification, thus adding hybrid materials and tissue preparation strategies into the HAM. ${ }^{11,12}$

Despite the unique characteristics of HAM as a wound dressing, there are still significant limitations in delivering complete amniotic membranes to irregular or large defects. This is due to the fact that, in most treatment cases, the amniotic membrane does not conform to the irregular topology or size of the defect. ${ }^{7}$ In addition, due to the high cost of processing, transportation, and storage of living cellulosic tissue, the clinical applications of HAM have been limited. It has been reported that there are anti-angiogenesis and angiogenesis characteristics derived from the epithelial and mesenchymal layers of $\mathrm{HAM}^{13}$ Decellularization not only eliminates the immunogenicity of HAM, but also exposes its extracellular matrix proteins, ultimately improving its biological characteristics. ${ }^{13,14}$

In recent years, a variety of hydrogel biomaterials have been widely used in tissue engineering, including as carriers for proteins, drugs and living cells. ${ }^{7,15,16}$ In the meantime, some researchers have successfully assembled dHAM and the fibroin protein produced by electrostatic spinning into threedimensional double-layer artificial skin. ${ }^{11}$ dHAM coated with polyester polyurethane on both sides, to improve its biomechanical strength and to produce a biocompatible surgical mesh structure, has also been reported. ${ }^{17}$ Van den Bulcke et al. reported a GelMA synthesis protocol in 2000. ${ }^{18}$ Because of its excellent performance and the preparation of hydrogels that avoid the use of a chemical cross-linking agent, GelMA has been widely used in different biomedical applications and proven to have good biocompatibility. ${ }^{19}$ Photolithography and threedimensional printing technologies can be used to fabricate micro-patterned 3D cell-laden GelMA hydrogels containing single or multiple cell lines for engineering different tissues. ${ }^{20}$ Through a detailed study of GelMA characteristics, our research group also clarified a series of properties for GelMA, marking it as an ideal biomaterial for tissue engineering. ${ }^{21,22}$

In this paper, to overcome the disadvantages associated with HAM grafting alone, we present a new composite class of semisynthetic scaffold for the clinical management of oral mucosal defects. The scaffold is a photo-cross-linked GelMAdecellularized HAM particle composite (GelMA-dHAP), processed into cell-free products while maintaining a high concentration of dHAP-derived extracellular matrix proteins. Although ultraviolet light has been extensively used in the optical cross-linking of different biopolymers, it has been reported to be associated with DNA and tissue damage. ${ }^{23,24}$ In addition, Lai found that the duration of ultraviolet irradiation had a great influence on the biological stability and matrix permeability of photo-cross-linked AM materials. ${ }^{25}$ Here, we describe for the first time the engineering of composite the GelMA-dHAP scaffold through visible light-mediated photocross-linking. The use of a photoinitiator system activated by visible light eliminates the biosafety issues associated with UV light, while yielding mechanical properties similar to or better than those of UV-cross-linked hydrogels. ${ }^{26}$ In this paper, we show that dHAP and GelMA can be effectively cross-linked into a biocomponent, 3D bulk structure scaffold that resulted in better biomechanical properties while preserving the desired properties of the original dHAM components (Fig. 1).

\section{Materials and methods}

\section{HAM collection and decellularization}

Pregnant women were screened serologically for the possibility of infectious diseases, such as human immunodeficiency virus type II, hepatitis B virus, hepatitis C virus, syphilis, gonorrhea, toxoplasmosis, cytomegalovirus, etc. After informed consent of the donors, fetal membrane was removed by aseptic operation. The chorionic tissue was removed by blunt separation and the amniotic layer was retained. All of the procedures were performed according to Mazaher et al. (2015). ${ }^{13}$ The fresh placenta was rinsed with sterile distilled water three times. Residual blood was discarded from the placenta and washed several times with sterile distilled water. HAM was isolated from the chorionic layer and transferred to sterile phosphate-buffered saline (PBS) ( $\mathrm{pH}$ 7.4) and treated with antibiotics and antifungal agents. The HAM was then treated in $0.2 \%$ EDTA for $30 \mathrm{~min}$ at $37^{\circ} \mathrm{C}$, and, thereafter, with $0.5 \mathrm{M}$ sodium hydroxide for $30 \mathrm{~s}$. The tissue was then transferred to $5 \%$ ammonium chloride and shaken vigorously. The cells of the HAM were removed by vigorous shaking and scraping and then washed with sterile PBS three times. The D tissue (dHAM) was lyophilized and pulverized using a tissue grinder (Tissuelyser-64, Jingxin, Shanghai, China) to form dHAP. The dHAP was sealed with nylon and preserved at $-80{ }^{\circ} \mathrm{C}$ until further use. All steps were performed under aseptic conditions. An overview of this process is shown in Fig. 1.

\section{Synthesis of methacrylated gelatin (GelMA)}

GelMA was synthesized using the methods described previously. ${ }^{21,22}$ Type A gelatin extracted from porcine skin was mixed in $10 \%(\mathrm{w} / \mathrm{v})$ PBS at a constant temperature of $60{ }^{\circ} \mathrm{C}$ with stirring, until the gelatin was completely dissolved. Methyl methacrylate (MA) was dropped into the gelatin solution over a period of one hour. After the addition of MA, the reaction solution was covered with aluminum foil to avoid light and reacted in darkness for 3 hours. After dilution with $40{ }^{\circ} \mathrm{C}$ PBS, five times, the reaction stopped. Then, dialysis was performed with 10-14 kDa cut-off membranes for one week to remove unreacted MA and other salts from the solution. After dialysis for one week, the solution was vacuum filtered via a membrane with a pore diameter of $0.22 \mu \mathrm{m}$. The filtered solution was then lyophilized to obtain a white spongy foam, GelMA. The foam was stored in a desiccator until needed. 


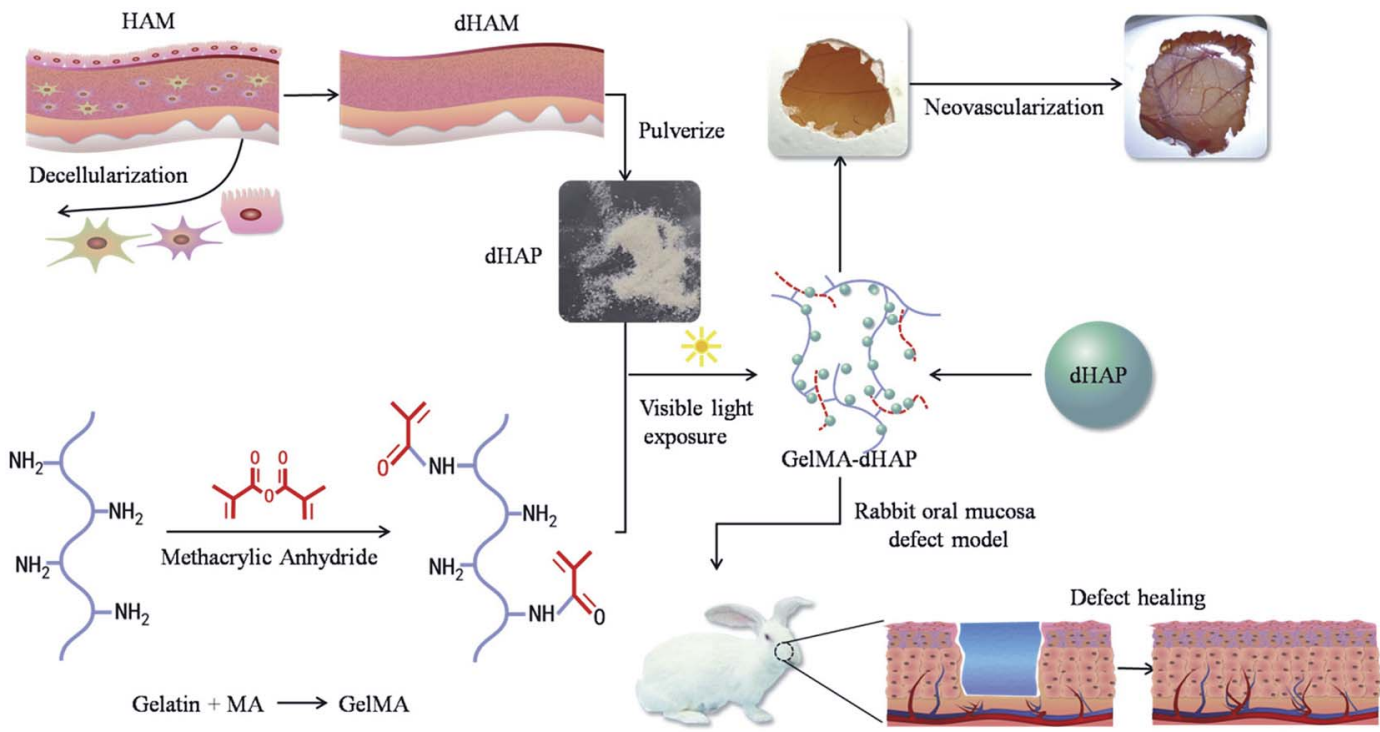

Fig. 1 Schematic preparation of the 3D porous structure scaffold, angiogenic potential of the GelMA-dHAP on CAM and efficacy of GelMAdHAP as a wound treatment on the rabbit oral mucosa defect model. HAM: human amniotic membrane; dHAM: decellularized human amniotic membrane; dHAP: decellularized human amniotic particles; CAM: chick chorioallontoic membrane.

\section{Preparation of dHAP-GelMA bicomponent 3D bulk structure scaffold}

GelMA foam (400 mg) and dHAP (200 mg) were prepared. The GelMA foam was dissolved in PBS to obtain GelMA solution with a concentration of $10 \%(\mathrm{w} / \mathrm{v})$, and dHAP was added and stirred evenly. Acylphosphinate photo-initiator (AP, L0290, Tokyo Chemical Industry, Japan) at $0.1 \%(\mathrm{w} / \mathrm{v})$ was added to the solution as a photoinitiator. Following addition of L0290, the solution was mixed and exposed to a visible light, dental curing device (Ultradent Products, 395-480 nm, $10.5 \mathrm{~mm}$ curing tip) for $20 \mathrm{~s}$ to form a $0.2 \mathrm{~cm}$ thick membrane. The synthesized GelMA-dHAP scaffold was washed with PBS and kept at $-80{ }^{\circ} \mathrm{C}$ until further use. The schematic of the GelMA-dHAP oral mucosa scaffold preparation is illustrated in Fig. $1 .^{27}$

\section{Surface characterization using scanning electron microscopy} (SEM)

GelMA-dHAP was taken from the $-80{ }^{\circ} \mathrm{C}$ refrigerator, lyophilized and sputter-coated with $\mathrm{Au}-\mathrm{Pd}(2 \mathrm{~nm})$ and examined with an S-4800 scanning electron microscope (Hitachi, Japan). The SEM was used to evaluate the surface of the freeze-dried scaffold and to achieve image collection. A total of 100 pores were randomly selected to measure their diameters.

\section{Fourier-transform infrared (FTIR) spectroscopy of GelMA, dHAM and GelMA-dHAP}

The molecular conformations of GelMA, dHAM and GelMAdHAP were determined by FTIR spectroscopy (ThermoFisher, Nicolet 6700). ${ }^{28}$ For this purpose, the dried GelMA, dHAM and GelMA-dHAP powders were mixed with potassium bromide $(\mathrm{KBr})$ and analyzed in the spectral region $4000-400 \mathrm{~cm}^{-1}$, with a resolution of $4 \mathrm{~cm}^{-1}$.

\section{Mechanical testing}

A universal tensile strength tester at a cross-head speed of 10 $\mathrm{mm} \min ^{-1}$ with a specified sample size (length $=26 \mathrm{~mm}$ and width $=10 \mathrm{~mm}$; stress $=$ force/[3× thickness $]$; strain $=$ length $/$ 26) was used to measure the biomechanical behavior of dHAM,
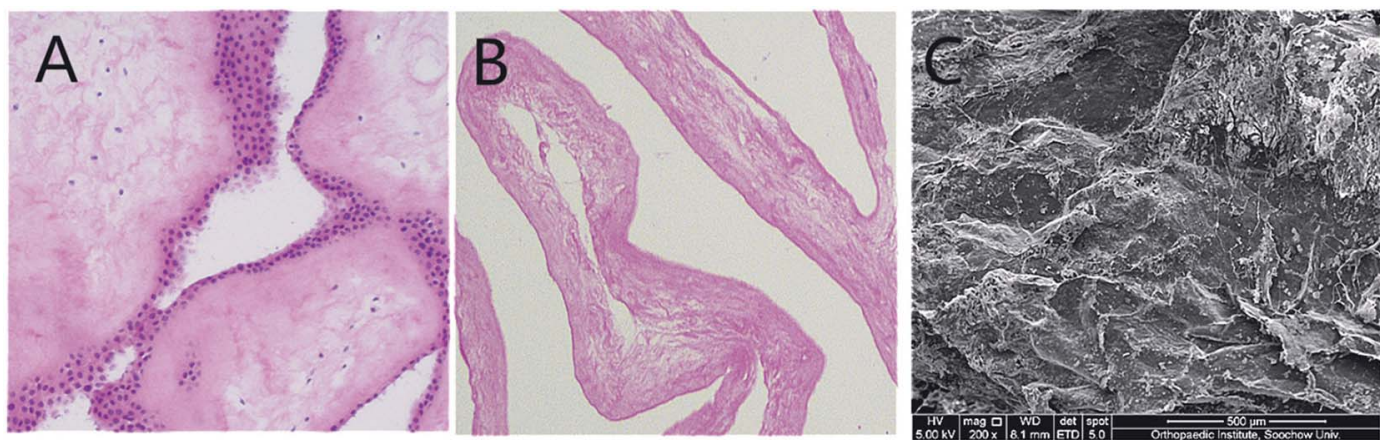

Fig. 2 (A) HAM before decellularization. (B) HAM after decellularization, H\&E staining showed that there was no blue stain on the acellular matrix surface. (C) Under scanning electron microscope, acellular matrix showed no residual cells, while fibrous structures are observed. 
GelMA and GelMA-dHAP $(n=3)$. The ends of the samples were fixed with a metal clip and then subjected to a force gradient of $0.25 \mathrm{~N} \mathrm{~min}^{-1}$, starting from a $2 \mathrm{mN}$ preload force until the sample fractured. The stress-strain curve was collected for each specimen, and the results for strain to fracture were calculated.

\section{Cytotoxicity assessment}

Cytotoxicity of GelMA-dHAP was assessed according to previous reports. ${ }^{29,30}$ Briefly, according to the ISO10993-5 standard, GelMA-dHAP composite was added into fresh cell culture medium at $6 \mathrm{~cm}^{2} \mathrm{~mL}^{-1}$ and incubated for $48 \mathrm{~h}$ at $37^{\circ} \mathrm{C}$ and the supernatants were collected as extracts. The extracts were diluted at volume ratios of $100 \%, 50 \%$, and $25 \%$ to produce a working solution, using fresh cell culture media. Meanwhile, cell culture medium was used as negative control and 5\% dimethyl sulfoxide as positive control. Human fibroblasts (HFF, Stem Cell Bank, Chinese Academy of Sciences) in logarithmic growth phase were digested and seeded on a 96-well plate at $10^{4}$ cells per $100 \mu \mathrm{L}$ per well at $37^{\circ} \mathrm{C}$ and $5 \%$ volume fraction of $\mathrm{CO}_{2}$ overnight. Then, each well medium was aspirated and $100 \mu \mathrm{L}$ extraction solution was added, $5 \%$ positive control using dimethyl sulfoxide, and continuing cultivation for $24 \mathrm{~h}$. Adding $100 \mu \mathrm{L}$ cck-8 reaction solution ( $90 \mu \mathrm{L}$ medium $+10 \mu \mathrm{L}$ cck-8) to each well and to the blank hole, as cck- 8 only, at $37^{\circ} \mathrm{C}$ for $2 \mathrm{~h}$ incubation, the absorbance of the culture medium was measured at $450 \mathrm{~nm}$. Each test group included six different experimental samples. Relative cell survival rate $=$ (experimental group A value - blank group A value)/(negative control group A value - blank group A value) $\times 100 \%$.

\section{Angiogenic potential of GelMA-dHAP}

The angiogenic potential of GelMA-dHAP was studied with a chick chorioallontoic membrane (CAM) assay. Specificpathogen-free (SPF) eggs were obtained from Beijing Technology Co., Ltd (China). GelMA, GelMA-dHAP and absorbable sponge with an internal diameter of $5 \mathrm{~mm}$ were prepared, with the absorbable sponge as the control group. At embryonic development day 4 (EDD), material from the same group was placed on the CAM surface at opposite sites. At EDD 4, $2.5 \mathrm{~mL}$
$4 \%$ paraformaldehyde fixing solution was added to the pseudochamber and fixed at room temperature for $15 \mathrm{~min}$. Using tweezers and scissors, the CAM film was removed from the window and placed in a Petri dish with normal saline. Then, the CAM film was stuck to the filter paper and photographs taken under the stereoscope. Thereafter, the CAM film was fixed in $10 \%$ formaldehyde, embedded in paraffin and $3 \mu \mathrm{m}$ sections were cut. According to a previous report, ${ }^{31,32}$ the total vascular area of all traced vessels was calculated by ImagePro Plus 6.0. After conventional hematoxylin and eosin (H\&E) staining, the vascular and inflammatory responses in the CAM were observed under a light microscope.

\section{In vivo oral mucosa repair}

New Zealand white rabbits $(2 \mathrm{~kg}$, male and female, animal service center of Suchow University) were randomly separated into three groups. Oral mucosal defects were covered with GelMA or GelMA-dHAP, with an oiled gauze dressing outer covering, while the control group was only covered with an oiled gauze dressing. There were four rabbits for each group, and eight samples per group (left and right sides of the oral mucosa were both sampled). The experiments were approved by the Soochow University Board for Animal Experiments. According to previous reports, ${ }^{33,34}$ the main observation indices were as follows (with one rabbit sacrificed in each group randomly, observing the rabbit general situation and the wound condition). (1) Changes to the buccal mucosa wound diameter: observed at days $3,5,7$, and 14 post-surgery. (2) Cell number and morphological observation of the defect repair site: observed at 3, 5, 7 and 14 days after surgery, the wound margin and normal wound tissue were fixed with $4 \%$ formaldehyde, and paraffin-embedded sections were stained with H\&E to observe morphological changes at the defect repair site.

\section{Statistical analysis}

Statistical analysis was performed using the GraphPadPrism7 program and SPSS 17.0, the mean between groups was analyzed using one-way analysis of variance (ANOVA), and the $q$ test (Newman-Keuls method) was statistically processed to
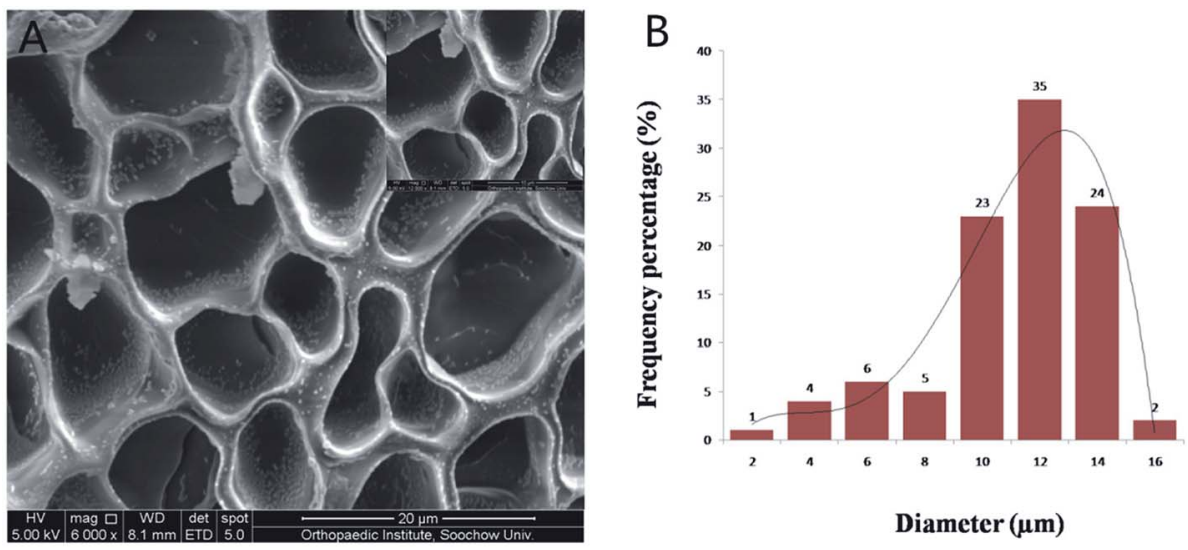

Fig. 3 (A) SEM image of pore size distribution in the composite GelMA-dHAP scaffold. The scaffold has a 3D porous network structure in which the dHAP are adhered and wrapped. (B) The distribution of pore size in the GelMA-dHAP scaffold. 


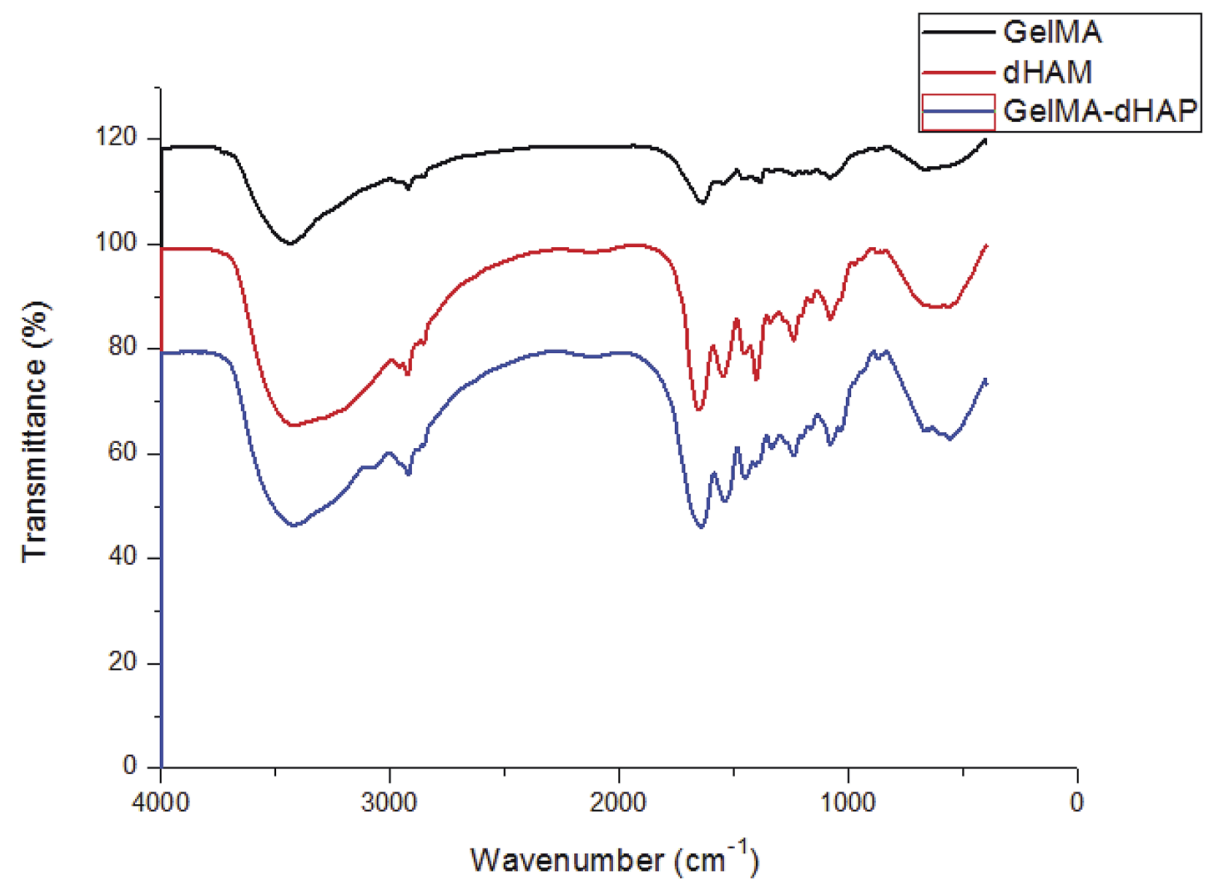

Fig. 4 The infrared spectra of GelMA, dHAM and GelMA-dHAP. No significant absorption peaks of new groups appeared in the GelMA-dHAP infrared spectra.

determine the significant differences. All values are expressed as mean \pm standard deviation. Differences were taken to be significant for $P<0.05$.

\section{Results}

\section{dHAM}

The decellularization of HAM was described previously. ${ }^{13}$ Complete removal of the cells and cellular debris was confirmed using H\&E staining (Fig. 2). H\&E staining showed that there was no blue stain on the acellular matrix surface. Under SEM, the acellular matrix showed no residual cells, while fibrous structures are observed.

\section{GelMA-dHAP characterization}

Morphology. The morphology of GelMA-dHAP was observed under SEM. The composite scaffold was obtained after the GelMA and DHAP were mixed and cross-linked with a visible light, dental curing device. The GelMA solution flowed freely before photo-cross-linking and transformed into a solid substance after cross-linking with dHAP. The 3D bulk structure of the GelMA-dHAP scaffold was shown to be highly porous (Fig. 3) by SEM. The pores are nearly round, with thin walls and uniform size. The average pore diameter is about $10.16 \pm 2.77$ $\mu \mathrm{m}$. Such a pore size and the porosity are very suitable for tissue engineering applications. ${ }^{31}$ The tilt view SEM image of GelMAdHAP confirmed coating of GelMA by dHAP.
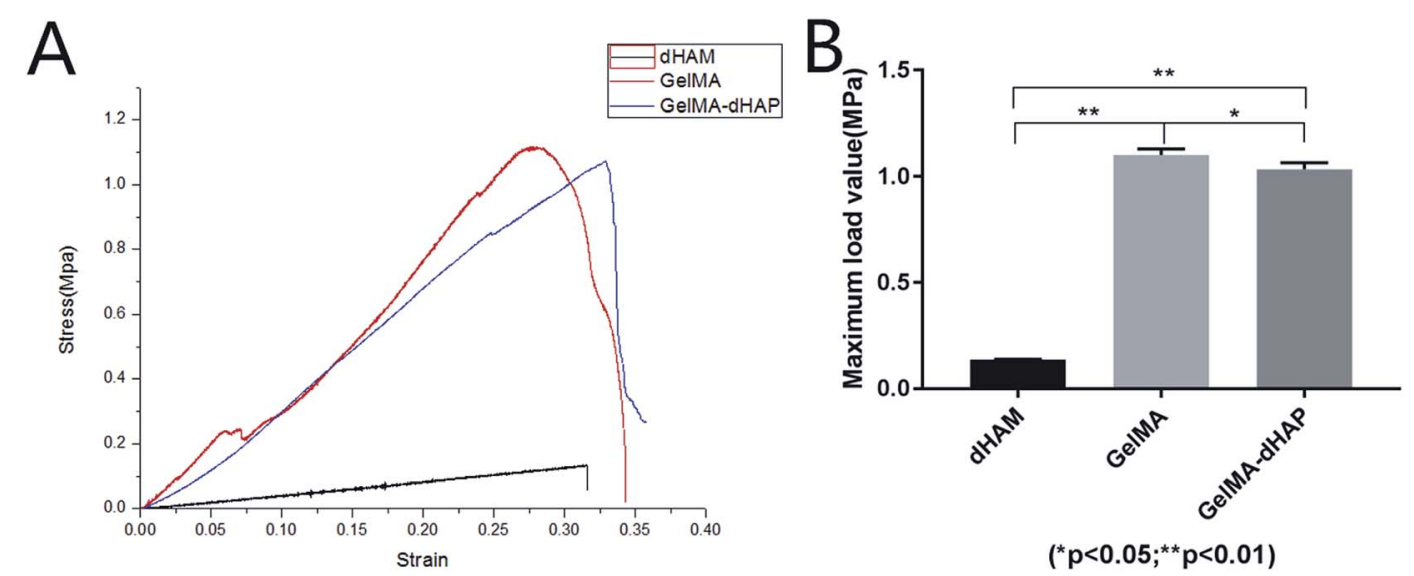

Fig. 5 (A) Stress-strain curves of dHAM, GelMA, and GelMA-dHAP. (B) Comparison of the maximum load value for dHAM, GelMA, and GelMAdHAP $(* P<0.05, * * P<0.01)$. 


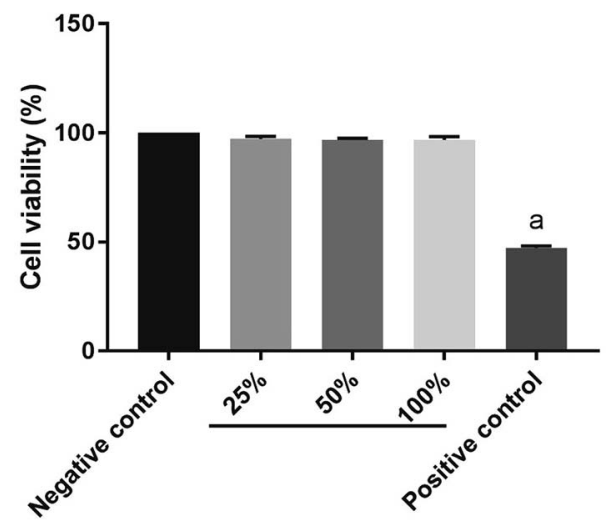

Fig. 6 Cytotoxicity: compared with the negative control group (a: $P<$ $0.05)$ and GelMA-dHAP showed no cytotoxicity.

\section{FTIR}

The conformational transitions of GelMA, dHAM and GelMAdHAP were confirmed by FTIR spectroscopy. The infrared spectra are depicted in Fig. 4. Compared with GelMA and dHAM, no significant absorption peaks for new groups appeared in the GelMA-dHAM infrared spectra.

\section{Biomechanical behavior}

In tensile testing, GelMA-dHAP showed significantly improved mechanical properties at maximum load value when compared with dHAM samples (Fig. 5). The composite scaffolds were stable until a tensile load of $1.04 \pm 0.03 \mathrm{MPa}$. The biomechanical testing in this study showed that GelMA-dHAP could significantly improve its mechanical properties which may make it an excellent 3D substitute for oral mucosa tissue engineering applications.

\section{Cytotoxicity assessment}

The cytotoxicity of GelMA-dHAP on HFF was evaluated according to the ISO10993-5 standard. Earlier relevant observations have shown that neither dHAM nor GelMA affected the cell viability. ${ }^{\mathbf{1 3 , 2 1}}$ Here, no cytotoxicity of GelMA-dHAP on the growth of HFF was detected (Fig. 6).

\section{The angiogenic potential of GelMA-dHAP}

Macroscopic observation showed that the number of neovascularizations was significantly increased on CAM for the GelMA-dHAP group, and the blood vessels were arranged as spokes, while the control group and GelMA group showed less vascular growth on CAM (Fig. 7A). The angiogenesis area was quantified by ImagePro Plus 6.0. The results showed that the angiogenesis area of the control group, GelMA group and GelMA-dHAP group were $23.33 \pm 1.59 \mathrm{~mm}^{2}, 22.95 \pm 1.82 \mathrm{~mm}^{2}$ and $33 \pm 0.81 \mathrm{~mm}^{2}$, respectively (Fig. 7B). Compared with the control group and GelMA group, the angiogenesis area of the GelMA-dHAP group was significantly increased $(P<0.05)$. H\&E staining of the GelMA-dHAP group showed that there was almost no inflammation in the CAM, whereas the control group and GelMA group showed mild inflammatory reaction with inflammatory cell infiltration around the blood vessels (Fig. 7C).

\section{In vivo oral mucosa repair}

No significant post-operative wound infection or positive allergy reaction were found in any of the groups. H\&E staining showed
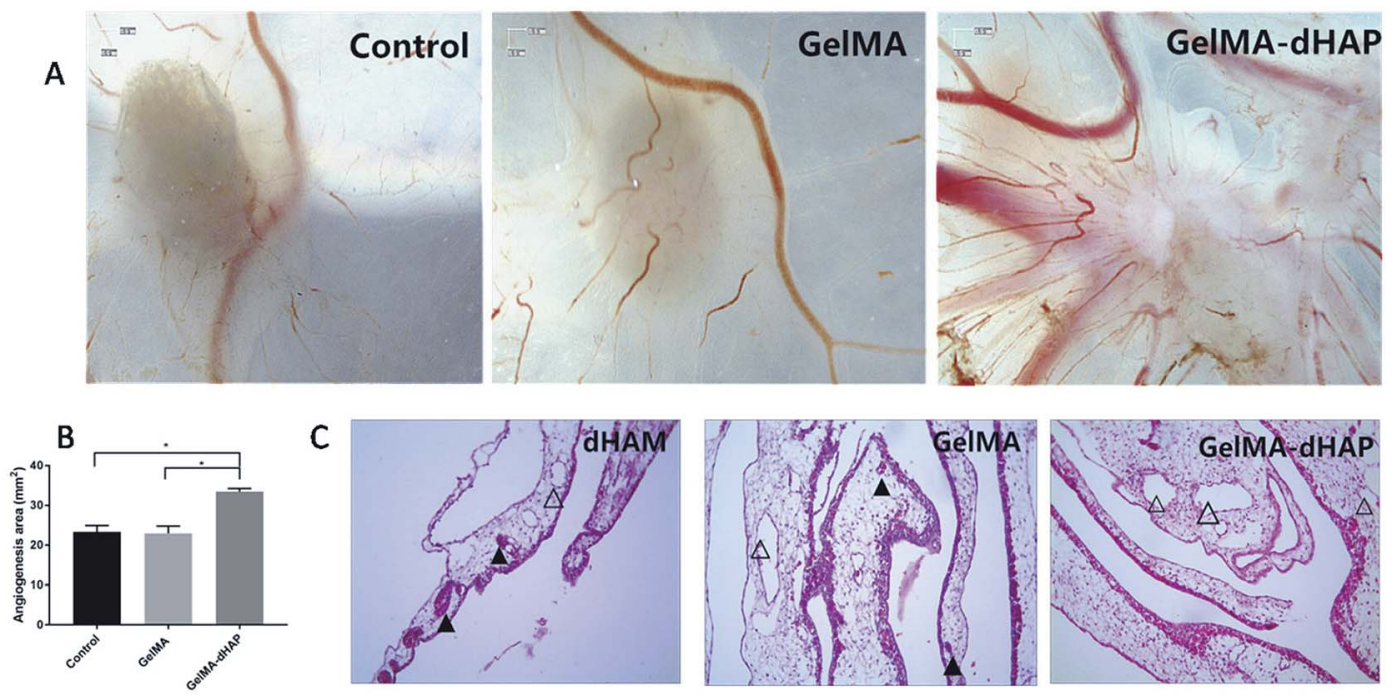

Fig. 7 Evaluation of the angiogenic properties of GelMA-dHAP in the chick chorioallantoic membrane (CAM) assay. (A) Micrograph stereoscopy images of the control, GelMA and GelMA-dHAP groups taken on day 8 of embryonic development. (B) The change in total angiogenesis area of control, GelMA and GelMA-dHAP group calculated from stereoscopy images (*P<0.05). Scale bars: $0.5 \mathrm{~mm}$. (C) Representative images from each group demonstrating almost no inflammation in the CAM of the GelMA-dHAP group, whereas a mild inflammatory response, together with infiltration of inflammatory cells around the blood vessels, was observed consistently in the control group and GelMA group. Scale bars: $100 \mu \mathrm{m}$. The solid triangles indicate the presence of inflammatory cells in the blood vessels, and the hollow triangles indicate no inflammatory cell infiltration in blood vessels or less inflammatory cells. 

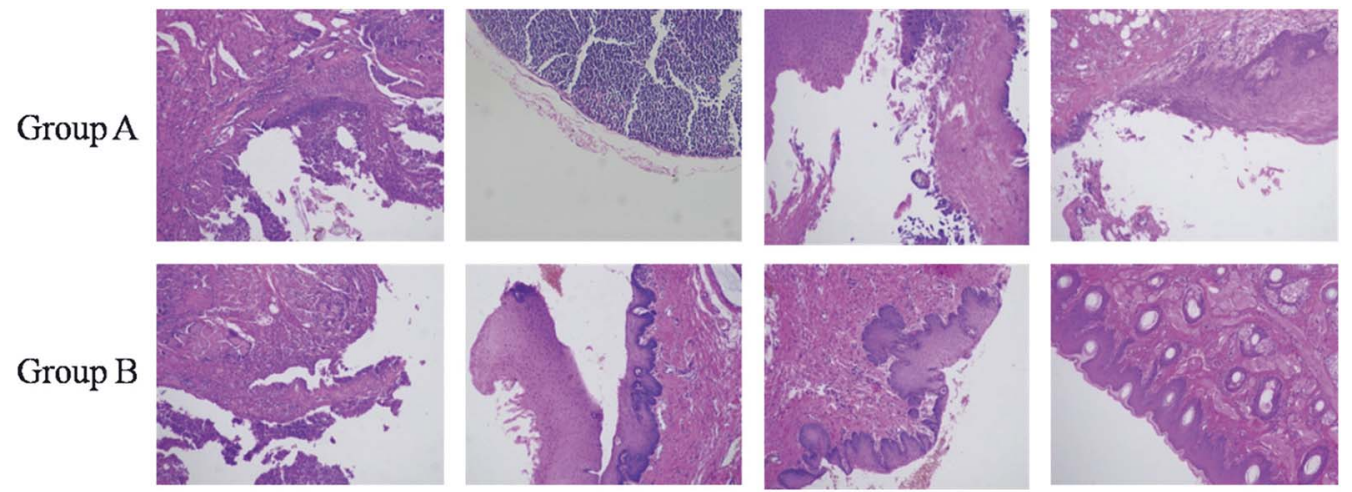

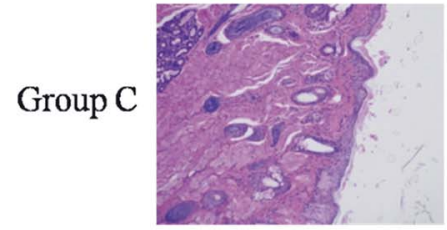

Day 3

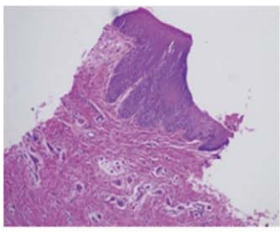

Day 5

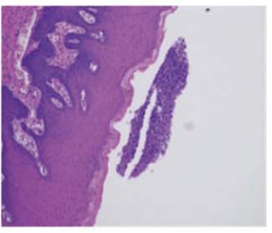

Day 7

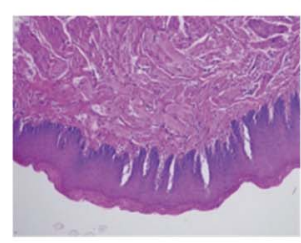

Day 14

Fig. 8 Histology of oral mucosa healing. H\&E staining photographs of oral mucosa wound on days 3, 5, 7, and 14. The oral mucosal defects were covered with oiled gauze in group A; GelMA in group B; and GelMA-dHAP in group C.

that $3,5,7$ and 14 days after the operation, new granulation tissue was found to be rich in capillaries, inflammatory cells and fibroblasts at the margin of the GelMA-dHAP operation area. The GelMA group showed a small amount of newborn epidermal cells covering the wound surface, with uneven thickness and cell swelling. In the control group, there were a large number of inflammatory cells and fibroblasts in the new granulation tissue, but few in the new epidermis. At 14 days after surgery, epithelial nappes were found in the operation area of the GelMA-dHAP group, while the epithelial cell layers were gradually increased in the GelMA group, with more fibroblasts and inflammatory cells. The number of inflammatory cells was relatively high in the control group, collagen fibers were disordered, and epithelium formation was poor (Fig. 8).

\section{Discussion}

Although HAM has good regeneration ability, its high storage cost and relatively weak mechanical strength greatly limits the application of fresh and dehydrated HAM. ${ }^{9}$ Tissue engineering scaffolds must provide sufficient biomechanical properties for better application in the regeneration of defect tissue. HAM and dHAM are difficult to graft due to their poor mechanical properties. To solve this problem, researchers are trying to find a way to improve the biomechanical behavior of HAM without affecting its characteristics, such as cell adhesion and noncytotoxicity. M. Gholipourmalekabadi et al. ${ }^{11}$ developed a three dimensional silk fibroin/amniotic membrane wound dressing with excellent chemical and mechanical properties for biological applications. In addition, the biomechanical properties of HAM were improved by cross-linking carbodiimine and glutaraldehyde. However, the negative effects of these drugs on the structure, molecular stability and cellular compatibility of HAM have been confirmed previously. ${ }^{35,36}$

In the present study, we synthesized and characterized a novel photo-cross-linking composite scaffold of GelMA-dHAP which has been designed as a dHAM substitute for oral mucosa repair. The composite scaffold combined the advantages of dHAM and GelMA. The photo-cross-linking between the GelMA and the dHAP generated a 3D porous structure that, not only drastically improved the biomechanical properties of dHAM, but also retained the bioactivity of dHAM. Due to dHAM being rich in proteoglycan and various growth factors, and to avoid DNA damage from ultraviolet light, the main findings of this study are that one can readily combine dHAP with GelMA to synthesize a 3D bulk scaffold using only $20 \mathrm{~s}$ of visible light cross-linking. The GelMA-dHAP is non-cytotoxic and promotes angiogenesis, which in turn promotes the repair of oral mucosal defects.

The microenvironment of the extracellular matrix plays an important role in cell morphology, adhesion, expansion and differentiation. Similar to the effects promoted by different biochemical cues, many biomimetic scaffolds have been developed with specific physical properties and topographic cues to influence the behavior of cells. Previous studies have shown that GelMA is a biocompatible material, which can be cross-linked in vitro to form a physical barrier. ${ }^{21,22}$ At present, previous studies have confirmed that amniotic membrane apoptosis does not affect the composition of the amniotic extracellular matrix. ${ }^{37}$ It is well known that amniotic epithelial cells and stromal cells have immunogenicity, so graft rejection restricts the use of HAM as an allograft. It has also been demonstrated that dHAM has many favorable characteristics, such as antibacterial properties, low antigenicity, ready availability and positive effects on cell proliferation and 
adhesion. ${ }^{13,37}$ The GelMA-dHAP scaffold serves as a dynamic environment extracellular matrix, and its $3 \mathrm{D}$ porous structure is easy to manipulate, which is of great significance for future scaffold handling in the laboratory and in the clinic.

\section{Conclusion}

The wide use of HAM for wound care has been shown to be safe and effective, yet it is not fully satisfactory for oral mucosa defect repair. This is because of its fast rate of biodegradation and low non-optimal biomechanics. In this study, we developed and fully characterized a 3D porous oral mucosa substitute scaffold made from dHAP and GelMA. The GelMA-dHAP scaffold shows improved mechanical properties when compared with dHAM alone. In in vivo animal experiments, the composite scaffold can promote repair of oral mucosal defects within two weeks. However, this approach to achieving a functional threedimensional porous scaffold is still worthy of further study with respect to the sterilization of GelMA-dHAP, autologous or donor cell research, biodegradability and swelling capacity studies. In addition, before moving from laboratory to clinical applications, it is also necessary to make sure that the scaffold can be translated into a suitable product in order to test it in humans. The present study has established the initial foundation for a novel and practical approach in the area of oral mucosa substitute research. We conclude that the GelMA-dHAP assessed in this study appears to be very promising when combined with seed cells as an approach to providing a wellvascularized 3D porous scaffold to improve the engraftment of tissue-engineered oral mucosa.

\section{Ethical statement}

All experiments were performed in compliance with relevant laws or guidelines and followed Soochow University institutional guidelines. All HAM was obtained from the discarded placenta and informed consent was obtained from the pregnant woman. All animal procedures were performed in accordance with the Guidelines for Care and Use of Laboratory Animals of Soochow University and approved by the Animal Ethics Committee of Soochow University.

\section{Conflict of interest}

The authors declared that there was no conflict of interest in the financial and publication of the work. All the authors gave their seal of approval to the manuscript.

\section{Acknowledgements}

This work was supported by the National Natural Science Foundation of China (K112222616 and 81671028), Shanghai Municipal Education Commission-Gaofeng Clinical Medicine Grant Support (20171906) and Shanghai talent development fund (2018099).

\section{References}

1 P. Agrawal, S. Soni, G. Mittal and A. Bhatnagar, Int. J. Lower Extremity Wounds, 2014, 3, 180-190.

2 F. Piraino and S. Selimovic, Mol. Cell. Ther., 2015, 2015, 1-10.

3 J. Yu, T. R. Huang, Z. H. Lim, R. Luo, R. R. Pasula, L. D. Liao, S. Lim and C. H. Chen, Adv. Healthcare Mater., 2016, 23, 2983-2992.

4 G. Ram-Liebig, J. Bednarz, B. Stuerzebecher, D. Fahlenkamp, G. Barbagli, G. Romano, U. Balsmeyer, M. E. Spiegeler, S. Liebig and H. Knispel, Adv. Drug Delivery Rev., 2015, 181-191.

5 J. C. Rigal-Satourne, J. M. Legeais, J. M. Texier, M. Savoldelli, J. P. Renard, J. F. Maurin and G. Renard, Invest. Ophthalmol. Visual Sci., 2000, 4, S455.

6 J. Y. Lai and L. J. Luo, RSC Adv., 2015, 5, 3425-3434.

7 S. V. Murphy, A. Skardal, L. J. Song, K. Sutton, R. Haug, D. L. Mack, J. Jackson, S. Soker and A. Atala, Stem Cells Transl. Med., 2017, 11, 2020-2032.

8 A. A. Mohammadi, S. M. Seyed Jafari, M. Kiasat, A. R. Tavakkolian, M. T. Imani, M. Ayaz and H. R. Tolideie, Burns, 2013, 2, 349-353.

9 K. G. Cornwell, A. Landsman and K. S. James, Clin. Podiatr. Med. Surg., 2009, 4, 507-523.

10 N. G. Fairbairn, M. A. Randolph and R. W. Redmond, J. Plast. Reconstr. Aesthet. Surg., 2014, 5, 662-675.

11 M. Gholipourmalekabadi, A. Samadikuchaksaraei, A. M. Seifalian, A. M. Urbanska, H. Ghanbarian, J. G. Hardy, M. D. Omrani, M. Mozafari, R. L. Reis and S. C. Kundu, Biomed. Mater., 2018, 3, 035003.

12 T. Nakamura, K. Endo, L. J. Cooper, N. J. Fullwood, N. Tanifuji, M. Tsuzuki, N. Koizumi, T. Inatomi, Y. Sano and S. Kinoshita, Invest. Ophthalmol. Visual Sci., 2003, 1, 106-116.

13 M. Gholipourmalekabadi, M. Mozafari, M. Salehi, A. Seifalian, M. Bandehpour, H. Ghanbarian, A. M. Urbanska, M. Sameni, A. Samadikuchaksaraei and A. M. Seifalian, Adv. Healthcare Mater., 2015, 6, 918-926.

14 A. K. Riau, R. W. Beuerman, L. S. Lim and J. S. Mehta, Biomaterials, 2010, 2, 216-225.

15 X. Zhao, X. M. Sun, L. Yildirimer, Q. Lang, Z. Y. Lin, R. L. Zheng, Y. G. Zhang, W. G. Cui, N. Annabi and A. Khademhosseini, Acta Biomater., 2017, 49, 66-77.

16 J. Y. Lai and Y. T. Li, Biomacromolecules, 2010, 5, 1387-1397. 17 P. N. Shi, M. N. Gao, Q. X. Shen, L. Hou, Y. B. Zhu and J. Wang, Mater. Sci. Eng., C, 2015, 54, 112-119.

18 A. I. Van den Bulcke, B. Bogdanov, N. De Rooze, E. H. Schacht, M. Cornelissen and H. Berghmans, Biomacromolecules, 2000, 1, 31-38.

19 K. Yue, G. Trujillo-de Santiago, M. M. Alvarez, A. Tamayol, N. Annabi and A. Khademhosseini, Biomaterials, 2015, 73, 254-271.

20 B. Byambaa, N. Annabi, K. Yue, G. Trujillo-de Santiago, M. M. Alvarez, W. T. Jia, M. Kazemzadeh-Narbat, S. R. Shin, A. Tamayol and A. Khademhosseini, Adv. Healthcare Mater., 2017, 16. 
21 X. Zhao, Q. Lang, L. Yildirimer, Z. Y. Lin, W. G. Cui, N. Annabi, K. W. Ng, M. R. Dokmeci, A. M. Ghaemmaghamin and A. Khademhosseini, Adv. Healthcare Mater., 2016, 1, 108-118.

22 W. Wu, Q. Ni, Y. Xiang, Y. Dai, S. Jiang, L. P. Wan, X. N. Liu and W. G. Cui, RSC Adv., 2016, 95, 92449-92453.

23 S. J. Bryant, C. R. Nuttelman and K. S. Anseth, J. Biomater. Sci., Polym. Ed., 2000, 5, 439-457.

24 N. Annabi, D. Rana, E. S. Sani, R. Portillo-Lara, J. L. Gifford, M. M. Fares, S. M. Mithieux and A. S. Weiss, Biomaterials, 2017, 139, 229-243.

25 J. Y. Lai, Mater. Sci. Eng., C, 2014, 45, 313-319.

26 S. L. Fenn and R. A. Oldinski, J. Biomed. Mater. Res., Part B, 2016, 6, 1229-1236.

27 N. Monteiro, G. Thrivikraman, A. Athirasala, A. Tahayeri, C. M. Franca, J. L. Ferracane and L. E. Bertassoni, Dent. Mater., 2018, 3, 389-399.

28 M. Gholipourmalekabadi, M. Bandehpour, M. Mozafari, A. Hashemi, H. Ghanbarian, M. Sameni, M. Salimi, M. Gholami and A. Samadikuchaksaraei, Burns, 2015, 7, 1488-1497.
29 K. X. Deng, X. Ye, Y. Y. Yang, M. Liu, A. Ayyad, Y. L. Zhao, Y. Y. Yuan, J. Z. Zhao and T. Xu, Neurol. Res., 2016, 9, 799808.

30 H. Liu, Z. Zhou, H. Lin, J. Wu, B. Ginn, J. S. Choi, X. Jiang, L. Chung, J. H. Elisseeff, S. Yiu and H. Q. Mao, ACS Appl. Mater. Interfaces, 2018, 17, 14559-14569.

31 G. Eke, N. Mangir, N. Hasirci, S. MacNeil and V. Hasirci, Biomaterials, 2017, 129, 188-198.

32 N. A. Lokman, A. S. F. Elder, C. Ricciardelli and M. K. Oehler, Int. J. Mol. Sci., 2012, 8, 9959-9970.

33 J. Tang, Y. Han, F. Zhang, Z. Ge, X. Liu and Q. Lu, Int. J. Artif. Organs, 2015, 2, 105-112.

34 Z. Ge, Q. Yang, X. Xiang and K. Z. Liu, Int. J. Oral Surg., 2012, 5, 673-680.

35 D. H. Ma, J. Y. Lai, H. Y. Cheng, C. C. Tsai and L. K. Yeh, Biomaterials, 2010, 25, 6647-6658.

36 E. Spoerl, G. Wollensak, F. Reber and L. Pillunat, Ophthalmic Res., 2004, 2, 71-77.

37 M. Gholipourmalekabadi, M. Sameni, D. Radenkovic, M. Mozafari, M. Mossahebi-Mohammadi and A. Seifalian, Cell Proliferation, 2016, 1, 115-121. 